\title{
Le Long voyage du Père Lycan
}

\section{Michel Coclet}

En irrespectueux hommage à un tas de gens.

Lorsque 1e Père Lycan ouvrit les yeux qu'il avait fermés quelques heures auparavant, il s'aperçut qu'il ne voyait rien. Deux hypothèses se présentèrent à son esprit, et l'esprit en question, après les civilités d'usage, les examina de la tête aux pieds, c'est-à-dire fort attentivement. Ces deux hypothèses, avouons-le, étaient loin d'être séduisantes; on peut même dire sans exagération aucune qu'elles étaient laides comme des poux. Toujours est-il que le Père Lycan s'absorbant dans 1 'examen de ces foutues hypothèses se mit à soliloquer comme suit: "Sacré nom d'un petit bonhomme! Comment-est-ce que ça se fait-il que j'y voyons rien? De deux choses l'une: ou bien $j$ 'ons perdu la vue subito presto, ou bien j'ons z'été percipité dans un grand trou tout noir, muni d'un couvercle étanche, et sans musique, sacré nom d'un petit bonhomme!"

Il faut dire pour la défense du Père Lycan qu'il était fort tard, et que par voie de conséquence les petits rouages délicats de son cerveau se refusaient obstinément à fonctionner comme ils eussent dû. Ils crissaient, grinçoyaient et crachouillaient, en sales petits rouages fainéants et mal élevés qu'ils ẻtaient; I'un d'eux alla même jusqu'à émettre un ricanement sarcastique autant que déplacé à $l^{\prime}$ adresse de la'moins séduisante des hypothèses, puis passa en roue libre sans plus de façons, de sorte que tout le mécanisme se mit à tourner à vide. Et le Père Lycan se reprit à soliloquer interminablement: "Sacré nom d'un petit bonhomme, etc, etc. . . . . " 
Quand le destin cruel au doigt crochu, à l'ongle noir et aveugle agrippe sa victime, quand le sort maléfique accable le miséreux tel un marteau pilon qui s'abat à coups redoublés, force brute et sans âme, symbole de notre civilisation mécanisée (où le matériau humain est pilé et fondu dans le grand creuset à l'instar du mil ou du sarrasin, transformé en galette et jeté en pâture aux perles et aux pourceaux), et quand le violoniste se fait Parthe (comprenne qui peut!), c'est triste.

Le petit rouage voyou et mal élevé était toujours en roue libre, coinçant la bulle sur son axe et, pour employer le langage des sciences physiques, se complaisant fort dans un équilibre indifférent. L'indifférence, heureusement, n'était point partagée par le reste des rouages qui commençaient à la trouver mauvaise. Quoi? Ce rebelle lunatique allait-il se permettre encore longtemps de troubler l'ordre? Ce propre-à-rien présentait un réel danger pour tout le mécanisme. Qu'il s'avisât de rester à l'écart une ou deux heures de plus, et les dégâts seraient irréparables. Déjà 1 'huile commençait à baisser dans le fond du carter, déjà les engrenages des niveaux supérieurs privés du précieux lubrifiant émettaient de sinistres grincements, đéjà le mouvement se ralentissait (ne confondons pas énergie cinétique et mouvement perpétue1); la terrible rouille ne tarderait pas à faire son apparition redoutée, à ronger, à corroder, en un mot à détruire. Bien sûr, il y avait toujours la possibilité de recourir à la mécano-chirurgie, mais en dépit de l'incontestable progrès que la récente découverte du degrip-oil permet dans ce domaine, les traumatismes post-opérationnels restent toujours quasi irrésorbables. De plus, 1'exemple de ce trublion était contagieux, I'anarchie rampait, un vent de fronde se levait, et ce n'était pas contre le Mazarin que ce vent là grondait. L'esprit du Père Lycan retentissait d'un tumulte inexprimable: au ronron de tous les jours s'ajoutaient les grincements des rouages privés d'huile, les récriminations des engrenages consciencieux réduits à I'oisiveté, les sifflements aigus du vent de fronde dans le mécanisme, et par dessus tout, la voix du Père Lycan qui soliloquait imperturbảblement: "Sacré nom d'un petit bonhomme! Comment-est-ce que ça se faiti1. . . etc. . . etc. . ." 
Ce pauvre Père Lycan était dans un état lamentable: de quoi émouvoir le plus insensible des rouages (un rouage au carbure de tungstène par exemple). I1 n'avait jamais été ce qu'il est convenu d'appeler un brillant sujet, mais tant qu'il s'appliquait à raisonner, si lentement que ce fût, il faisait encore bonne figure. Et même quelques instants après que le mécanisme de son esprit ait commencé à tourner à vide, son monologue était resté parfaitement compréhensible, grâce à la vitesse acquise, à la force de 1 'habitude, et un observateur non averti n'aurait rien pu déceler d'anormal. Il n'en était plus de même à présent: au fur et à mesure que le trouble se répandait en son âme, ses paroles devenaient confuses, son élocution indécise, pâteuse même, les mots s'entrechoquaient, se cabossaient, se déformaient, se recollaient comme ils pouvaient, c'était affreux. On en arrivait peu à peu mais inéluctablement à de sourds grognements inarticulés, entrecoupés de hoquets et de longs éclats de rire hystériques qui se prolongeaient et s'amplifiaient en résonnant à l'intérieur du crâne oblong du Père Lycan.

Cela affectait de plus en plus le fonctionnement déjà fortement compromis du délicat mécanisme logé dans ce crâne; et plus le mécanisme en était affecté, plus les éclats de rire se faisaient fréquents. Il y avait maintenant trois petits rouages qui avaient suivi $1^{\prime} \mathrm{e}-$ xemple du factieux, cause de tous les ennuis; les dernières gouttes d'huile s'écoulaient par le conduit d'évacuation; la fin était proche. C'est alors qu'en un élan sublime, faisant preuve d'une abnégation héroĨque, tout le mécanisme s'allia en un effort communautaire contre les éléments perturbateurs afin de les réduire et de les juguler, au risque de se perdre. Tous les rouages voisins des rebelles se déplacèrent sur leurs axes autant que le jeu nécessaire au bon fonctionnement le leur permettait, les axes eux-mêmes se déformèrent jusqu'à la limite de rupture pour permettre aux engrenages qu'ils supportaient d'atteindre les dissidents, et ceux-ci, pris entre deux et parfois trois engrenages, furent obligés de rentrer dans le rang et de se soumettre à la volonté générale. Le mécanisme était en márche. Une clameur, faible et assourdie d'abord, mais de plus en plus forte, une clameur d'allégresse s'éleva 
par dessus $\bar{l}$ e ronronnement qui reprenait, la pompe à huile se remit en route, et le premier moment de délire passé on n'entendit plus que le chant joyeux du syndicat des roues, pignons et engrenages: "Dent pour dent, dent pour dent. Dent pour dent, dent pour dent". L'alerte avait été si chaude que tout ce petit peuple laborieux, mais dont certains membres s'étaient laissé entraîner sur le chemin de la négligence et de la nonchalance, mettait à présent tout son coeur à la tâche, avec une ardeur qui faisait plaisir à voir. Jamais cela n'avait si bien marché.

Le Père Lycan ouvrit les yeux qu'il avait femés quelques heures auparavant, et $s^{\prime}$ aperçut de nouveau qu'il ne voyait rien. Mais il se sentait extrêmement bien; en fait, il ne s'était jamais si bien senti. Et lorsque deux hypothèses se présentèrent à son esprit, il leur fit un petit signe guilleret de la main et les examina comme s'il n'avait fait que cela de son existence. Plus de soliloque infiniment répété et qui ne mène qu'au désastre, plus d'élocution indécise. Il se sentait en pleine possession de ses moyens. I1 commença par classer les deux hypothèses en deux catégories. Il rangea dans la première les circonstances qui déperdaient de lui, et dans la seconde celles qui ne dépendaient pas de lui. Cette classification opérée, le Père Lycan entreprit fort logiquement d'examiner chacun des êléments du problème. Si l'hypothèse selon laquelle il avait perdu la vue s'avérait être la bonne, il n'y avait rien qu'il pût faire dans l'immédiat; cela relevait directement du domaine de la médecine ou de 1a chirurgie, voire de la mécano-chirurgie; et bien que le Père Lycan ait éprouvé dès sa plus tendre enfance un intérêt extrêne pour ces trois sciences -- eu égard à leur caractère humanitaire -- son échec au Certificat d'Etudes Primaires $I^{\prime}$ avait empêché de se consacrer, ainsi qu'il l'eût souhaité, à I'étude d'abord et par suite à $1^{\prime}$ exercice de la médecine. Toujours est-il qu'à quelque chose malheur est bon, car à supposer que le père Lycan ait eu des notions précises en ces diverses sciences, il eût été à craindre qu'il ne s'avisât de s'opérer lui-même sur le champ, avec des instruments de fortune, dans des conditions d'asepsie quasi inexistantes, courant ainsi à un échec certain. Son absence de connaissances médicales lui permit donc d'éliminer sans 
plus balancer la première hypothèse et de passer à la seconde.

Et pendant ce temps-là les petits rouages tournaient à plein rendement, avec un doux ronronnement, sans heurt et sans reproche, conscients de la noblesse de leur tâche, et ce sentiment du travail bien fait les aidaient à accepter de bonne grâce les vicissitudes de leur sort, à surmonter les moments passagers de découragement dus à la fatigue. On entendait de temps à autre des gouttes d'huile excédentaires qui tombaient de toute la hauteur du mécanisme, et leur flic-floc était aux engrenages ce que les chants d'oiseaux sont aux cantonniers; on entendait aussi parfois un gros soupir émis par 1'un des ex-trublions, mais aussitôt suivi de "Tss! Tss! Tss!" à la fois réprobateurs, compatissants, encourageants et paternels, provenant des rouages voisins qui veillaient à la rééducation des dissidents et les aidaient à réintégrer leur place dans la société. Dans la pénombre, tout ce petit peuple laborieux oeuvrait conscien cieusement.

En examinant 1a seconde hypothèse, le Père Lycan se dit: "Sacré nom d'un petit bonhomme! Si que j'ons z'été percipité dans un grand trou tout noir, muni d'un couvercle étanche et sans musique, n'y a pas à paniquer. Sauf mon respect, je me battions $I^{\prime}$ oeil que $n^{\prime} y$ a point de musique! N'y a personne qui pourrera m'accuser d'aller plus vite que les violons! Et à plus vite que j'irions, à plus vite que je serions sorti de mon hypothétique trou noir! Refléchissons, que diable, refléchissons!" Et ce faisant, il apparut au Père Lycan que si le trou était, comme il le supposait, muni d'un couvercle étanche, il lui fallait porter ses efforts sur cet élément du problème. Qui dit couvercle étanche dit couvercle; qui dit couvercle dit cooperculum et qui dit cooperculum dit couvrir. Il ne manquait plus au Père Lycan que le préfixe "dé" pour sortir de son trou. Aussitôt, méthodiquement, et avec une agilité que nul n'eût soupçonnée, le Père Lycan se mit à fouiller ses poches, à les passer au peigne fin, dans l'espoir d'y trouver ce qu'il y cherchait. Sur le sol, près de lui, s'empilait une foule d'objets hétéroclites, tels qu'il s'en trouve dans toutes les poches. Ayant accompli cette besogne exténuante, le Père Lycan s'assit sur sa chaise 
dans l'ombre et contemplant avec satisfaction le petit préfixe qu'il tenait au creux de sa main, il se mit une fois de plus à soliloquer, mais en des termes cette fois fort étranges: "Zфgxuk tekhitkswat fethekk $\phi$ ! Gekkstw $\phi-$

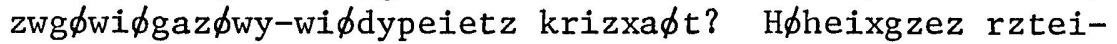
faф . . . $\phi_{w k a} . . . \emptyset_{w k a} . . . \emptyset_{w k a} . . . "$

L'esprit du Père Lycan était le théâtre d'une horreur rarement égalée, non point tant par son caractère spectaculaire que par son insidiosité. Dans l'ombre, silencieusement, sournoisement, I'hurluberlu factieux en faisait de belles: comme le Père Lycan s'acheminait lentement vers $1 \mathrm{a}$ fin de ses ennuis, au moment même où il mettait la main sur la clé du problème, ce malotru d'engrenage sans foi ni loi, ce misérable bout de ferraille était en train de commettre un acte défiant toutes les lois de la morale rouagique la plus élémentaire. Comme il ne pouvait plus ni s'arrêter, ni se mettre en roue libre, il venait de changer de langage! O horreur! Il s'était branché sur le poldave! Il tournait certes. mais poldave! Et le Père Lycan, parlant poldave, n'avait que faire de ce ridicule préfixe "dé" qu'il tenait dans ses mains. Il lui aurait fallu à présent la particule séparable " $\phi n$ ", et il ne suffit pas de parler poldave pour se retrouver ipso facto avec un assortiment complet de toutes les particules séparables de la langue poldave dans les poches! Et cette saleté de petit rouage polyglotte qui continuait à tourner poldave, et à ricaner silencieusement poldave de toutes ses dents.

Le Père Lycan alors se hissa sur ses pieds, laissa choir tristement son préfixe inutile, et l'écrasa par terre d'un coup de savate traînard et qui manquait de conviction. C'en était trop. A quoi bon! Telles étaient les profondes considérations auxquelles se laissait aller le Père Lycan. Et pour commencer, se disait-il en poldave, on peut très bien vivre dans un trou! Les mineurs par exemple, et sans couvercle même. $D^{\prime}$ accord ils ont un ascenseur et plusieurs étages. Ici pas d'ascenseur, mais c'est de plain-pied. Un peu bas de plafond peut-être. Et levant le bras pour se faire une idée des dimensions de son nouveau domicile, le Père Lycan n'eut aucune difficulté à toucher le plafond. I1 éprouva même une certaine surprise en se rendant 
compte qu'il lui suffisait de faire jouer les muscles de sa main, de ses doigts, de ses phalanges, pour que le plafond cédât sous la pression; et cela lui procurait une vive satisfaction physique. Le Père Lycan s'arrêta soudain en se disant qu'il pouvait peut-être réserver 1'usage ludique de ses muscles pour des activités ultérieures, et passer sans plus tarder aux applications pratiques du principe qu'il venait de découvrir. Il s'apprêtait à mettre à exécution cette pensée lorsque le couvercle s'ouvrit brusquement, sans grincer, mais en s'exclamant: "Eh! Jules! Ben $v^{\prime} l a$ aut' chose! Et sur une nationale! Viens voir ici!.. . Jules? Jules! Ben où qu'il est?" Puis il se tut.

Et 1à-dessus le Père Lycan se remit à penser en français, comme tout le monde. Le choc avait été trop grand pour le petit rouage (ou le rouage trop petit pour le choc): n'ayant jamais rencontré de couvercle parlant auparavant, il en oubliait de tourner poldave, tant ce phénomène le troublait. De temps à autre, comme il tentait de chasser de ses pensées cet inquiétant mystère, il revenait au poldave, puis re-tournait français, et ainsi de suite. Cela présentait un léger inconvénient pour le Père Lycan, car les transitions d'une langue à $1^{\prime}$ autre s'effectuaient avec une lenteur désespérante, de sorte que pendant de longues minutes, tout ce que le Père Lycan pouvait penser et dire se résumait à ces quelques termes: "Ah goch! Ah goch, goch, goch!" qu'il ne pouvait s'empêcher de noyer dans des flots de salive. Pendant les intervalles où sa pensée était claire, le Père Lycan se posait une foule de questions. Qu'était-ce que ce Jules? Pourquoi n'avait-il pas répondu à I'appel de son nom? Quand allait-il le faire? Ne serait-il pas judicieux de sortir de ce trou? Le Père Lycan établissait lentement une relation encore mal définie entre la venue proche de Jules et son sort personnel, et commençait à se douter que 1'un pourrait fort bien affecter $1^{\prime}$ autre. Tandis qu'il s'efforçait de formuler ce doute avec toute la clarté et la concision dont il était capable (condition nécessaire et suffisante pour passer à l'action), une scène d'une importance capitale se déroulait à la surface autant qu'à son insu.

La voix qui avait tant surpris le petit rouage anar- 

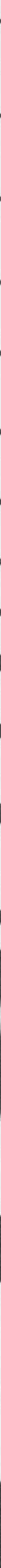
chico-polyglotte n'était certes pas venue du couvercle, mais d'un individu qui s'éloignait maintenant à grands pas à la recherche de Jules, vraisemblablement afin de lui relater verbalement les faits qu'il venait de constater, et de lui exposer son opinion en la matière. Il espérait sans doute de la sorte inciter Jules à se transporter sur les lieux dont s'agit, lui faire partager la même expérience, et, par la comparaison de leurs opinions respectives, fonder leur jugement de façon solide et certaine. Toujours est-il qu'il progressait avec une rapidité inhabituelle sur le bas-côté de la route, son corps perdant parfois tout contact avec la terre et s'élevant dans les airs, mais pour retomber ensuite et rebondir aussitôt, le même pied ne touchant jamais le sol deux fois de suite: il courait. I1 courait à la rencontre de Jules dont il apercevait la mince silhouette adossée à un poteau télégraphique distant d'une centaine de mètres; il arrêta sa course vagabonde en arrivant à la hauteur de ce poteau, et entreprit la silhouette en des termes d'une éloquence rare. Dès que Jules eut compris ce dont il était question, il se coinça un vieux mégot dans la bouche d'un geste décidé, remonta le pantalon de son bleu de travail, fit faire à sa casquette un demi-tour complet qui amena la visière par derrière, et se dirigea vers un curieux engin garé au bord de la route.

I1 est inutile de disstinuler flus longtemps la véritsable identité de Jules et son compagnon: ils étaient tous deux cantonniers de première classe, récemment promus au 3éme échelon, ce qui leur donnait droit à une augmentation de salaire non négligeable, au titre de grands manoeuvriers du destin affectés à l'exécution des basses oeuvres, et au port des insignes afférents à ce titre, sans mentionner les avantages secondaires et tertiaires. En vertu de ces avantages, ils étaient autorisés à faire fonction de gardiens du patrimoine national, dans un de ses aspects qui pour ne pas être des plus nobles n'en est pas pour autant moins nécessaire: ils étaient chargés de la réfection des routes nationales jusques et y compris la peinture des lignes jaunes et blanches, médianes et latérales, sous 1a haute direction du Ministère des Travaux Publics. La présence d'un trou, si beau fût-il, au milieu de $1 a$ 
route nationale, constituait une anomalie qui relevait donc directement de leur compétence et les deux manoeuvriers s'apprêtaient à y mettre bon ordre sans plus tarder. Jules avait en effet décidé de faire siennes les opinions de son collègue, et décliné l'invitation de celui-ci d'aller constater de visu, personnellement, et en la compagnie du collègue en question, le phénomène sus-mentionné. Ils s'étaient réparti les tâches fort équitablement, se proposant d'agir comme suit: Jules, usant du privilège que lui conférait $I^{\prime}$ ancienneté, se chargerait de diriger .l'E.P.L.J. (engin à peindre les lignes jaunes) tandis que son collègue comblerait le trou de gravillons goudronnés, ces délicates opérations devant être effectuées dans 1 'ordre normal des choses, la première d'abord et la seconde ensuite, contrairement à ce qui vient d'être indiqué.

Le Père Lycan avait pendant ce temps traversé plusieurs crises de logochée successives entre lesquelles il avait compris la nécessité urgente où il se trouvait de quitter ses pénates sub-nationales; il avait également considéré les diverses modalités de l'action qu'il envisageait d'entreprendre, et s'engageait à présent dans la première phase du processus d'extraction. I1 se tenait debout sur sa chaise et s'agrippait des deux mains aux rebords de son trou. Le piteux spectacle; cela faisait peine à voir: le coup de talon initial dont le but principal était de propulser le Père Lycan hors de son trou avait fait long feu', comme l'eût pu faire celui d'Achille après $1^{\prime}$ accident. Le second coup de talon ne valait guère mieux, et avait été suivi immédiatement par une avalanche de coups de talons successifs et précipités, le Père Lycan ayant de toute évidence sacrifié l'esthétique du geste sur l'autel de l'efficacité.

Jules avait enfourché 1'E.P.L.J. qui gronchognait maintenant, tressaillait dans un immonde fracas, vomissait des flots de fumée malodorante par un orifice prévu à cet effet, et, râclant le sol de ses pneus, s'ébranlait lentement. Le collègue de Jules, quant à lui, s'était placé dans les brancards d'une super-brouette emplie de gravillons goudronnés et la traînait derrière lui; il dépassa le trou de quelques mètres puis souleva I'avant de son véhicule, ce qui eut pour conséquence 
immédiate de faire baisser l'arrière. La brouette se trouvant alors, si $1^{\prime}$ on peut dire, en pente, le gravilIon commença à s'écouler.

Le Père Lycan jaillit hors de son trou en ouissant 1 'horrificque crépitement et s'étala de tout son long, face contre terre. Il se releva dès qu'il fut en mesure de ce faire, pour apercevoir le collègue de Jules qui proféra ces mots horribles: "Ben, mon cochon!" Le Père Lycan ne fit ni une ni deux (ni trois ni quatre). Un tonnerre monstrueux rugissait derrière lui: le soleil se couvrait d'une brume jaunâtre. I1 se tourna d'un coup et vit 1'E.P.L.J.

Alors, figé sur place, il se sentit aspiré irrésistiblement par le monstre, qui l'engloutit dans sa gueule béante aux senteurs de fuel-oil. Il perdit conscience. Quelques secondes plus tard, après avoir été broyé et mélangé à la peinture bouillante, il était recraché par l'E.P.L.J., étalé au beau milieu de la route en une longue ligne jaune fumante qui prenait par endroits des teintes orangées. Seule tressaillait encore une bulle contenant le petit rouage qui eut ainsi la joie d'assister au triomphe de șa force maléfique; il contempla satisfait le long cadavre du Père Lycan, mais peu à peu la peinture se fit opaque et il ne vit plus rien. Et Jules et son collègue, leurs travaux terminés, s'adjugèrent quelques minutes de pause en attendant cinq heures.

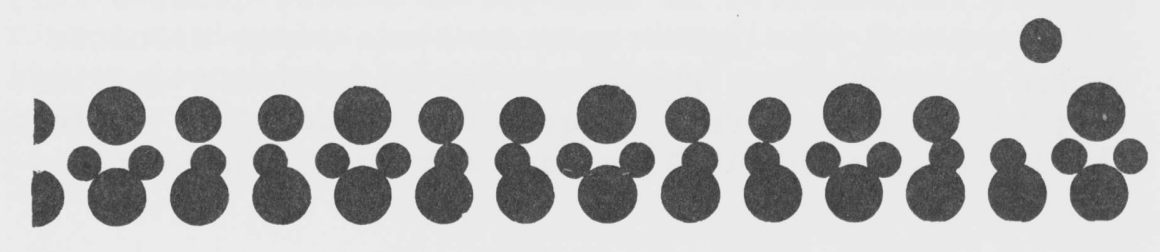

